This is a pre-publication draft. The final version of this paper was published in International Journal of Mathematics Education in Science and Technology, 42, 7, 867-878. Any citations should refer to that version.

\title{
Categorisation and analysis of explanatory writing in mathematics
}

\author{
Tracy S. Craig ${ }^{a, b 1}$ \\ ${ }^{a}$ Academic Support Programme for Engineering in Cape Town (ASPECT), \\ ${ }^{b}$ Centre for Research in Engineering Education (CREE) \\ University of Cape Town, South Africa
}

\begin{abstract}
The aim of this paper is to present a scheme for coding and categorising students' written explanations of mathematical problem-solving activities. The scheme was used successfully within a study project carried out to determine whether student problem-solving behaviour could be positively affected by requiring the writing of explanatory strategies to mathematical problem-solving processes. The rationale for the study was the recognised importance of mathematical problem-solving, the widely acknowledged challenge of teaching problem-solving skills directly and the evidence in the literature that writing in mathematics provides a tool for learning. The study was carried out in a first-year mathematics course at the University of Cape Town, South Africa. Students' written submissions were categorised and analysed through use of an adaptation of a journal entry classification scheme. The scheme successfully observed positive changes over the experimental period in students' level of engagement with the mathematical material and with their stance towards knowledge.
\end{abstract}

Keywords: problem-solving; writing; coding schemes; classification schemes

\section{Introduction}

The aim of this paper is to describe a scheme by which student writing in the context of mathematical problem-solving can be categorised and analysed using an adaptation of a journal entry classification scheme $[1,2,3]$. The classification scheme was used successfully within a study project located within a first year university mathematics course at the University of Cape Town, South Africa. The author had been involved for some years in lecturing a first-year mathematics course consisting primarily of calculus (differential and integral) as well as introductory complex numbers, vector geometry, linear algebra and infinite series. The course was compulsory for all students majoring in mathematical sciences, physical sciences, and actuarial science, and was a grounding course for many concurrent and future courses, including more advanced mathematics, applied mathematics, physics, chemistry, computer science, statistics, and financial mathematics. The syllabus for the course was very broad and contained much of an algorithmic and recipe-driven nature, albeit often cognitively challenging. Problem-solving, as strictly defined, was not taught in the course, and the occasional true problem (as opposed to exercise) encountered by the students in tutorials or tests was poorly approached, understood and performed.

Teaching problem-solving is an extremely challenging task, although it has been performed successfully in small classes with an emphasis on problem-solving [4]. Successful attempts to teach problem-solving very often include restructuring of a course, however, often involving a perceived decrease in course content. The research question addressed by the writing study project was whether problem-solving behaviour or ability might benefit from the writing of explanatory strategies without restructuring the existing and standard course, and, importantly, be discernable in the written submissions. To determine whether the intervention was successful in any way it was necessary (alongside simultaneous complementary analyses) to have a coding scheme by which any potential change in problem-solving behaviour could be

\footnotetext{
${ }^{1}$ Email: Tracy.Craig@uct.ac.za
} 
detected over the experimental time period. Problems, in this study project, were defined as non-routine mathematical questions, accessible to the students but with no immediate indication of solution strategy $[4,5]$. Problem-solving was implicitly defined as the solution of such problem, "problem-solving as skill" as defined by Stanic and Kilpatrick [6]. For a broad understanding of problems and problem-solving in mathematics see Schoenfeld [4, 7], Stanic and Kilpatrick [6] and Lester [8].

In this paper, a literature review of writing for learning in mathematics will be given, including writing for learning mathematics in general, writing for learning in problem-solving specifically and a review of reported studies drawing on data or grounded in theory. Next, the context of the use of the categorisation scheme in the writing study project will be described. The methodology section of the paper will discuss the coding scheme successfully adapted for use with the data and which is the object of interest in this paper. The results of the analysis are described and discussed, illustrating the success of the coding scheme. Finally the paper is concluded with implications for further investigations and the strengths and limitations of the study.

\section{Conceptualising writing for learning Writing for learning}

"Writing represents a unique mode of learning - not merely valuable, not merely special, but unique. ... Writing serves learning uniquely because writing as processand-product possesses a cluster of attributes that correspond uniquely to certain powerful learning strategies" [9: 122]. Why should writing be such a useful tool in mathematical learning? What "cluster of attributes" of writing supports cognitive development? The characteristics cited most frequently for the utility of writing are its permanence, its active nature and its pace $[9,10,11,12]$, all characteristics which are innately part of the writing process, and all interact to make writing a powerful tool for the learning process in general. In addition, there are characteristics which are arguable but which tend to be taken as similarly incontestable, such as the necessity of making connections, of being clear and explicit, and of the personal involvement in the process $[9,10,11,13,14,15]$. A final, yet enthusiastic, line of argument in favour of writing in mathematics is its perceived similarity to problem-solving in particular, rather than to any other form of learning. In reference to problem-solving and expository writing, Bell and Bell [11] suggest that if "their underlying processes are so very similar, practice in one area can reinforce competency in both by strengthening the student's critical thinking ability" [11:213]. The dialogue between mathematical problem-solving and expository writing deserves special consideration.

\section{Writing as and for problem-solving}

The support of writing as a successful technique of teaching mathematics in general, and mathematical problem-solving in particular, extend from the vague "the act of writing helps clarify both the process and content of problems" [16] to the intense "writing is problem solving" [5, emphasis in original]. It is further argued that writing encourages not only cognitive learning, but the acquisition of metacognitive skills [5, 13]. Glynn and Muth [17] describe the writing process in terms that are immediately reminiscent of competent problem-solving behaviour.

... competent writers ... can juggle several skills concurrently, interactively and fluidly ... Thus, while producing text, a competent writer also may be revising ideas or relations among ideas. It is important to note that all the writing skills interact with one another ... and are coordinated by the writer's metacognition. The writer who recognises that the produced text, even after revision, still does not achieve his or her goals, might then revise the plan for meeting those goals ... 
This is a pre-publication draft. The final version of this paper was published in International Journal of Mathematics Education in Science and Technology, 42, 7, 867-878. Any citations should refer to that version.

[17: 1065]

It has been argued that writing is problem-solving, that thoughtful writing practice employs the identical cognitive processes as successful problem-solving, and thus is an ideal tool for use within a problem-solving context $[5,10,18]$. Indeed, writing and problem-solving are felt to enhance one another, rather than the effect only going one way [11], with Shield \& Galbraith [19] citing Hamilton (1990) as linking the two activities through their shared "generative" nature. Kenyon [5] defines a writing exercise as involving the following stages: planning (attempting to understand, ideas are generated and organised), composition (ideas are translated into extended text), and revising (text is reviewed, redundancies are removed, clarification is increased). Similar breakdowns of the writing process are encountered in Cross [15] and Mendez and Taube [18], all reminiscent of Pólya's [20] problem-solving steps of understand the problem - devise a plan - carry out the plan - look back.

\section{In search of theory}

The literature on writing to learn in mathematics takes on a variety of forms. The distinctions between studies are not that clear, however. The perhaps weakest grounding in the literature for writing supporting learning in mathematics is found in papers that amount to lists of suggestions of types of writing to employ in the classroom, or recounting of apparently successful writing experiments with no data given in support $[5,18,21]$. Even this weak form of support has its uses, providing the reader with ideas and encouragement. Examples in the literature of studies citing data tend heavily towards the qualitative $[12,16,22,23]$ rather than quantitative [11], although some studies successfully combine the two forms of analysis [24, 15].

A third clear category in the relevant literature is that providing a theoretical viewpoint of the subject. Examples include Garofalo \& Lester [25] providing a detailed set of categories of metacognitive activity, based on metacognitive theory, a theory also drawn on by Glynn and Muth [17]. Pugalee [13] drew successfully on Garofalo and Lester's metacognitive framework to carry out a data-driven analysis of student writing. Gopen and Smith [26] suggest Reader Expectation Theory as a form of analysing student writing. Keys [27] provides a coding scheme for analysing written lab reports. Lew and Schmidt [28] designed an automated coding scheme using SPSS software linguistic classification techniques. They criticise the potential subjectivity of manual coding, although they acknowledge weaknesses of an automated system. Shield and Galbraith [19], suggesting that a reason for the scattered nature of the writing branch of mathematics education research is the lack of coding schemes, provide a broad brush coding scheme for differentiating between expository writing and student journal writing styles. Waywood's journal classification scheme [1], widely cited in the literature, was found to be highly applicable to the author's study project. Morgan [29] uses Critical Discourse Analysis and linguistic theory such as Halliday's concept of register to interrogate and interpret students' mathematical texts. Nückles et al. [30] use Cognitive Load Theory to critique the changing role of "prompts" in journal writing over an extended period of journal writing. Leonard et al. [24] drew on the expert-novice research to provide support for their successful writing intervention in physics. Emig [9] provides valuable early theoretical contributions. Finally, there are the review papers, of great value to the researcher attempting to get an overview of a field, but tending to blur the 
distinctions between data based studies and less formal contributions. Several review papers were found useful in this study [10, 14, 30, 32, 33].

\section{Context - the writing study project}

The topic of discussion in this paper is the coding scheme, not the entire study project; however a brief outline of the context of the study is appropriate. The students registered for the mathematics course attended one 45-minute morning lecture every day. Once a week, it was compulsory that each student attend one of a number of twohour afternoon tutorials. Tutorials were conducted in groups, with students working through a given sheet of exercises (and occasionally problems ${ }^{2}$ ). Tutors were present to help as needed. The author acted as tutor for three tutorial groups, two experimental and one control. For the purposes of the writing exercise analysis the two experimental groups ${ }^{3}$ were considered as one. The control group (carrying out the identical standard tutorial as the experimental groups simply with no writing exercises) served as a comparison when problem-solving behaviour and ability was determined through analysis of course assessment tasks. The control group served as potential (and ultimately positive) evidence that any perceived improvement in problem-solving ability was due to the writing exercises and not simply the tutoring environment. A total of 39 students in the combined experimental group took part in the study, producing a total of 155 written submissions. 29 students attended the control tutorial group. Following instructions, the students wrote explanatory paragraphs on their problem-solving procedures during the tutorials and submitted them to the tutor at the end of the session (similar to the "writing only" group in [15]). While students could feasibly have completed more than one submission, in practice this did not occur. No formal training in problem-solving occurred other than that routinely present in the course. The students received brief formative feedback in the form of the author's comments on their returned submissions. The writing exercises were not graded. No differences in approach or results were observed across language (30 English, 9 non-English main language) or gender (14 female, 25 male) subgroups; this lack of difference is quite possibly due to the small numbers involved.

\section{Methodology}

Waywood [1] describes a classification system for student journal entries (see also [3]). Journal entries were regarded as having the forms Recount, Summary ${ }^{4}$ or Dialogue, each of which reflects a particular stance towards learning. The three categories represent a hierarchy of cognitive complexity and depth of engagement with mathematical content. Waywood's classification system worked satisfactorily for analysing the writing exercises, although it had to be adapted due to the difference in

\footnotetext{
${ }^{2}$ Since the writing initiative was explicitly intended not to interfere with the standard running of the course, the tutorial sheets were not manipulated by the author to contain strictly defined problems. Most weeks there were no strict problems on the tutorial sheets, simply exercises, often of a challenging nature. The assigned "problems" were, in general, exercises that were considered to best benefit from a look-before-you-leap approach.

${ }^{3}$ The degree programmes of the students in the tutorial groups were varied. The cohort included students registered in the Commerce Faculty ( 3 different degree programmes), the Engineering Faculty (5 degree programmes), the Science Faculty (5 degree programmes) and 1 degree programme in the Humanities Faculty.

${ }^{4}$ Retaining Waywood's terminology the word "summary" is used in two contexts. Lowercase use represents conventional usage of the word whereas uppercase use refers to the coding scheme's category.
} 
This is a pre-publication draft. The final version of this paper was published in International Journal of Mathematics Education in Science and Technology, 42, 7, 867-878. Any citations should refer to that version.

contexts. Waywood's original [1] three classifications were concerned with the actions of summarising, collecting examples, questioning and discussing.

Original classification (drawn from [1]):

Recount - summarising means record, examples show how to get answers, questions relate to how to do things, discussion means talking about what happened. Recount mode is characterised by concrete things to be done, an emphasis on objective description and reporting of passive observation of objective knowledge.

Summary - summaries are about stating and organising, examples show how a mathematical procedure is applied, questions are about misunderstandings, leading to discussion, discussion is about forming an overview. Summary mode is characterised by utilitarian involvement, the recognition and ordering of important ideas and the integration of utilitarian knowledge.

Dialogue - summaries are about integrating, examples are paradigms, questions are about analysing and directing, discussion is about formulating arguments. This mode is characterised by recognition of the requirement to generate mathematics, learning being shaped by inquiries and a creative stance towards knowledge generation.

The students taking part in the author's writing study project were instructed to explain and justify their actions in solving mathematical problems, a slightly different requirement to Waywood's structured journal entries. A rephrasing of Waywood's classification scheme allows for the description of the following three categories of writing exercise.

Recount - The student is reporting what has happened, there is evidence of passive observation and simple recording of events. There is a focus on obtaining answers, explaining how things are done and talking about what has happened. Knowledge is seen as objective, the observer has to simply receive it.

All examples from student writing exercises (although typed) use the students' original words, spelling and punctuation. Editorial comments are in square brackets.

Example of Recount

Which one of the following improper integrals converges?
(A) $\int_{0}^{1} \frac{1}{(x-1)^{2}} d x$
(B) $\int_{-1}^{1} \frac{1}{(x+1)^{2}} d x$
(C) $\int_{0}^{\infty} \frac{1}{(x+1)^{2}} d x$
(D) $\int_{0}^{\infty} \frac{1}{(x-1)^{2}} d x$
(E) $\int_{-2}^{2} \frac{1}{x^{2}} d x$

If you just glance through the available solutions, does one appear most likely? If so, which one? Solve the problem, however you wish. Pretend you are explaining the problem to a puzzled fellow student. Write out (in words, as much as possible) how you solved the problem. Was your final answer the one you expected (if you did)? If not, can you explain why? ${ }^{5}$

"Well, I just kind of did what was in my notes. If the limit does not exist then the integral diverges. So if a limit can be solved and a value found then the integral does exist." (RT, week 8)

RT refers explicitly to simply following the instructions in her notes from lectures. The steps are followed without any explanation of what they might be representing symbolically. The terminology is not explained and the words "kind of" suggest the student is unsure of what to write for this problem as following the steps is seen as all

\footnotetext{
${ }^{5}$ In the interests of brevity, mathematical problems henceforth in the paper will be included without the writing instructions seen by the experimental groups in the study.
} 


\section{T.S Craig}

there is to the problem. The student positions herself as the passive recipient of knowledge.

Summary - Content is codified and organised. External facts are integrated into an internal system of knowledge. There is an attempt at providing an overview. A good attempt is made to generalise observations. Technical terms are not simply used, but are explained. Knowledge is seen as functional, and has to be integrated with what is already known.

\section{Example of Summary}

Find the orthogonal trajectories of the family of ellipses $2 x^{2}+5 y^{2}=C$, and sketch several members of each family.

"The slope at any point that satisfies the elipse-family equation is, by definition, the derivative of the equation.

Orthogonal trajectories are given by $-1 / \frac{d y}{d x}$ which yields the function $|x|^{5 / 2} k^{5}$ which

looks quite similar to my guess.

Basically when the derivative of the elipse family equals the inverse reciprocal of some functions derivative, that function gives the orthogonal trajectories map." (DC, week 7)

DC related the process by which the problem is solved, then explicitly indicates understanding of what the calculations are representing by rephrasing the process. He refers back to his expectation of the problem's solution, thereby indicating that he can interpret his algebraic solution in the broader context of the problem.

Dialogue - There is interaction between ideas. Content is integrated. Calculations are analysed and directed. Arguments are formulated, there are attempts to explain phenomena or contradictions. There is evidence of creative knowledge use. Student learning is shaped by enquiries. Knowledge is seen as something to be (re)created. Example of Dialogue

After Gauss reduction, the following augmented matrix was obtained.

$$
\left(\begin{array}{ccc|c}
1 & 0 & 5 & 2+k \\
0 & 1 & 3 & 5-k \\
0 & 0 & k(k-1) & k
\end{array}\right)
$$

For which values of $k$ does the system have

i.no solution

ii.infinitely many solutions?

"I was not sure how to tackle this question, but I realised that the rows in the matrix are in fact plains in $\mathbf{R}^{3}$. When plains intersect, the intersection points form an infinite line along this intersection, thus proving an infinite number of points (providing the planes are not limited in any way). Thus, if I could find a value for $k$ which would make the two planes equal (i.e. find their points of intersection), I might find a solution (providing my understanding of vectors is not flawed)

thus I put $2+k=5-k$ as subsequently solved for $k$. (desperate attempt)" (IS, week 9)

The example of Dialogue was deliberately included as an example where the student did not correctly answer the question. The solution process does not have to be correct to merit the Dialogue description (Cross [15] makes a similar observation). The category of Dialogue was applied to that final example for the creativity shown, the way the student argues for his solution attempt, and the way the student is learning as 
This is a pre-publication draft. The final version of this paper was published in International Journal of Mathematics Education in Science and Technology, 42, 7, 867-878. Any citations should refer to that version.

he proceeds. All spelling, grammar and punctuation in the examples above are as they were in the students' original writing.

Waywood's classification system was easily applied to the data, with very little ambiguity about the category to which each writing exercise belonged. Since each writing exercise was on the single topic of discussion of a particular problem-solving process, there were found to be no instances of submissions which could have different sections categorised differently. The categorisation of each of the writing exercises was independently verified by a senior and experienced colleague.

\section{Discussion}

The results are (unfortunately but unsurprisingly) not smooth with each student neatly progressing through the categories. Certain students showed little progression, with their submissions being categorised as Recount in a majority of the weeks of the study. Conversely, other students did show progression and their deepening understanding of mathematical problems was supported by other complementary forms of analysis. To indicate deepening level of engagement with the mathematics, an example of each of Recount, Summary and Dialogue is given for the same student.

Solve the differential equation $x^{2} y^{\prime}+5 x y+3 x^{5}=0$.

"We have to divide through by $x^{2}$ first to get $y^{\prime}$ on its own. We will then find the integrating factor and multiply through by it and then solve the linear differential equation" (LM2, week 7, Recount)

The steps of the process are recounted without explanations, reasons or evidence of understanding of meaning. The student does not see herself as particularly involved in the mechanics of problem solution, but rather as the passive applier of a process received from an authoritative source.

After Gauss reduction, the following augmented matrix was obtained.

$$
\left(\begin{array}{ccc|c}
1 & 0 & 5 & 2+k \\
0 & 1 & 3 & 5-k \\
0 & 0 & k(k-1) & k
\end{array}\right)
$$

For which values of $k$ does the system have

(i) no solution

(ii) infinitely many solutions?

"In order for us to get an answer that states $\rightarrow$ no solution we have to have a system that is inconsistent meaning on a diagram it represents 3 planes that have no common point of intersection. In terms of equations you'll have

$$
\begin{aligned}
& x+0 y+5 z=3 \\
& 0 x+y+3 z=4 \\
& 0 x+0 y+0 z=1 \leftarrow \text { any number }
\end{aligned}
$$

[more equations]

In other words you have to find values of $k$ that would give you zero on the left and no. on the right." (LM2, week 9, Summary)

The term "inconsistent" is defined in terms indicating the student can see the links between linear algebra and vector geometry. While LM2's explanation is interspersed with calculation, the process is described in terms that indicate understanding of what the symbols represent. The steps are not simply meaningless stages of an algorithm but are seen to have meaning in the context. 
Let $A=\left(\begin{array}{lll}1 & 1 & 1 \\ 1 & 1 & 0 \\ 2 & 1 & 0\end{array}\right), \quad B=\left(\begin{array}{lll}2 & 3 & 4 \\ 0 & 1 & 5\end{array}\right)$.

Find $A^{-1}$ and (if possible) matrices $X$ and $Y$ such that $A X=B$ and $Y A=B$. "I have to first calculate the inverse of A i.e $\mathrm{A}^{-1}$ by Gauss Reduction [matrix]. This is when you are Gauss Reducing A against the square identity and we want to get the same sq. identity on the left in the end. $\mathrm{A}^{-1}$ will be the matrix that appears on the right of the (end product) Gauss formula? i.e. [matrix]. Take that inverse $\mathrm{A}^{-1}$ and multiply out with it throughout the equation [equation] and then solve for X (IF POSSIBLE). What I mean is matrix multiplication only occurs if the column of $\mathrm{m}^{\mathrm{x}} \mathrm{A}$ is equal to the row of $\mathrm{m}^{\mathrm{x}} \mathrm{B}$ i.e. if $A$ is a $2 \times 2 \mathrm{~m}^{\mathrm{x}}$ then $\mathrm{B}$ has to be either a $2 \times 3$ or anything as long as the row is 2 . Therefore you have $(2 \times 2) \times(2 \times 3) \therefore$ your resulting matrix is will be a $2 \times 3$ [numeric values in the last two sentences were scored through and overwritten as the student sorted out the correct associations]." (LM2, week 10, Dialogue, text in square brackets represents editorial commentary or trimming of calculations.)

LM2 gives a detailed and functional description of how to invert a matrix and thereafter how to use it in a matrix multiplication calculation. Her explanation raises itself to the level of Dialogue by the way the student is learning as she goes along, rephrasing and correcting herself. The presence of the question mark also indicates that the student is not merely expressing her fully formed cognitive understanding, but is questioning her use of either the terminology or the process. The student is involved in the process of learning while engaged in the writing task.

Over the course of the semester there was movement of relative frequencies away from simple recounting of facts and towards explanations. There was an apparent inclination to think more about the processes involved in arriving at answers, rather than simply getting the answers. The scheme's description of a change in the stance towards learning, away from the student as passive observer of objective knowledge and towards the student as active engager in the use or creation of knowledge was understood to have been observed. ${ }^{6}$

While comparison with the control group is, by definition, impossible in the context of the writing exercises, comparison was possible in various analyses of assessment tasks the students were required to complete throughout the year. The one criterion on which there was noticeable divergence between experimental and control after the beginning of the writing intervention was that of understanding ${ }^{7}$ (see also $[34,28$, 15]). In a number of assessment tasks throughout the year students' success at completing problem-solving questions was measured on a number of variables (such as raw grade obtained, indication of understanding, apparent metacognitive control, flexibility of solution), with understanding of the problem showing noticeable improvement for the experimental group compared to the control group. The coding

\footnotetext{
${ }^{6}$ Detailed evidence of the development of problem-solving processes in the student cohort is beyond the scope of this paper. The reader should be aware, however, that the scheme described in this paper successfully and unambiguously measured progress which was verified by concurrent and complementary forms of analysis [35].

${ }^{7}$ In contrast to other research in writing to learn [30], no improvement was observed in metacognitive phenomena.
} 
This is a pre-publication draft. The final version of this paper was published in International Journal of Mathematics Education in Science and Technology, 42, 7, 867-878. Any citations should refer to that version.

scheme thus was easy to apply, but also indicated trends in the data that were verified by other forms of analysis which are unfortunately not within the scope of this paper ${ }^{8}$.

\section{Conclusions}

Waywood's journal entry classification scheme was successfully adapted for use in both categorising and analysing explanatory strategies written in the context of mathematical problem-solving. The success of the scheme was twofold, both in its applicability to the data and in its positive measurement of change. The scheme, once adapted from the summary-example-question-discussion format of Waywood's original to a more general format, was unambiguously and unproblematically applied to the data. The scheme, as applied to the data collected over the course of the semester, successfully measured change in students' engagement with the mathematics and their stance towards knowledge.

A strength of the scheme is its applicability to short pieces of writing. Longer pieces of writing might include different sections which could be categorised differently, a danger which was not applicable to the short pieces involved in this study. A further strength of the scheme was its underpinning focus on stance towards knowledge. This focus allowed for the unproblematic generalisation of the categories away from the original requirement of structured journal entries to more freeform explanatory writing and, I argue, avoids the potential ambiguity [28] offered by certain other coding schemes. A weakness of the scheme was the perceived large gap (similarly observed by [3]) between Recount and Summary, a gap which might likewise be found to exist between Summary and Dialogue on a different data set. Potential extensions of this study include extending writing activities to the entire class rather than tutorial groups within the class, embedding writing within more class activities than a weekly tutorial and grading the exercises or otherwise making them compulsory for the targeted student body [36]. Waywood's lexical measure of engagement [cited in 28], found to positively correlate with teacher assessment of journal entries, would be a potentially productive avenue of investigation.

The categorisation resulting from use of the scheme allowed for observation of the success of the writing initiative in developing deeper understanding of mathematical questions in the students and perceived improvements in problem-solving behaviour. Waywood's journal classification scheme was the best coding scheme the author could find when the need for such was realised, however its framing (grounded in its use for coding structured journal entries) was an inadequate fit to the data represented by the writing exercises in this study project. The adaptation of Waywood's scheme was found to be easy and unambiguous to use in the context and ultimately led to interesting observations, particularly in light of simultaneous complementary analyses of the same data set.

The use of the adapted scheme in coding free form writing in the form of explanatory paragraphs on problem-solving processes is apparently novel. Such coding and subsequent analysis could be extended to other forms of student writing such as unstructured journal entries, writing about mathematical concepts (as opposed to problem-solving explanations), reports or mathematical projects. It is believed that the

\footnotetext{
${ }^{8}$ This analysis took the forms of structured analysis of problem-solving assessment tasks as well as Piagetian investigation of student interview data.
} 
adapted coding scheme fills a niche in the field of the study of writing and mathematical problem-solving analysis.

\section{References}

[1] A.Waywood, Journal writing and learning mathematics, For the Learn. Math. 12, 2 (1992), pp. 34-43.

[2] A.Waywood, Informal writing-to-learn as a dimension of a student profile, Ed. Stud. Math. 27 (1994), pp. 321-340.

[3] D.J. Clarke, A.Waywood and M. Stephens, Probing the structure of mathematical writing, Ed. Stud. Math. 25 (1993), pp. 235-250.

[4] A.H. Schoenfeld, Mathematical Problem Solving, Academic Press, inc., Orlando, Florida, USA, 1985.

[5] R.W. Kenyon, Writing is problem solving, in Writing to Learn Mathematics and Science, P. Connolly and T. Vilardi, eds., Teachers College Press, New York 1989, pp. 73-87.

[6] G.M.A. Stanic and J. Kilpatrick, Historical perspectives on problem solving in the mathematics curriculum, in The Teaching and Assessing of Mathematical Problem Solving, R.I. Charles and E.A. Silver, eds., Lawrence Erlbaum Associates, Hillsdale, New Jersey, 1989, pp. 1-22.

[7] A.H. Schoenfeld, Learning to think mathematically: Problem solving, metacognition and sense making in mathematics, in Handbook of Research on Mathematics Teaching and Learning, D.A. Grouws, ed., Macmillan, New York, 1992, pp. 334-370.

[8] F.K. Lester, Musings about mathematical problem-solving research: 1970 - 1994, J. Res. Math. Ed. 25, 6 (1994), pp. 660-675.

[9] J. Emig, Writing as a mode of learning, Coll. Comp. \& Comm. 28, 2 (1977), pp. 122-128.

[10] A.N. Applebee, Writing and reasoning, Rev. Ed. Res. 54, 4 (1984), pp. 577-596.

[11] E.S. Bell and R.N. Bell, Writing and mathematical problem solving: Arguments in favour of synthesis, School Sci. Math. 85, 3 (1985), pp. 210-221.

[12] I.W. Gaskins, J.T. Guthrie, E. Satlow, J. Ostertag, L. Six, J. Byrne and B. Connor, Integrating instruction of science, reading and writing: Goals, teacher development, and assessment, J. Res. Sci. Teach. 31, 9 (1994), pp. 1039-1056.

[13] D.K. Pugalee, Writing, mathematics, and metacognition: Looking for connections through students' work in mathematical problem solving, School Sci. \& Math. 101, 5 (2001), pp. 236-245.

[14] N.F. Ellerton and M.A. Clements, $A$ research agenda for the "Writing Mathematics" movement, in Mathematics: Meeting the Challenge, M. Horne and M. Supple, eds., Mathematical Association of Victoria, Melbourne, 1992, pp. 153-159.

[15] D.I. Cross, Creating optimal mathematics learning environments: Combining argumentation and writing to enhance achievement, Int. J. Sci. \& Math. Teach. 7 (2009), pp. 905-930.

[16] R. Borasi and B. Rose, Journal writing and mathematics instruction, Ed. Stud. Math. 20 (1989), pp. 347-365.

[17] S.M. Glynn and K.D. Muth, Reading and writing to learn science: Achieving scientific literacy, J. Res. Sci. Teach. 31, 9 (1994), pp. 1057-1073.

[18] B. Mendez and S.R. Taube, Mathematics and writing: Linking problem solving and composing strategies, J. Coll. Read. \& Learn. 27, 3 (1997), pp. 108-118. 
This is a pre-publication draft. The final version of this paper was published in International Journal of Mathematics Education in Science and Technology, 42, 7, 867-878. Any citations should refer to that version.

[19] M. Shield and P. Galbraith, The analysis of student expository writing in mathematics, Ed. Stud. Math. 36 (1998), pp. 29-52.

[20] G. Pólya, How to Solve It, second edition, Princeton University Press, Princeton NJ, 1945.

[21] W.P. Berlinghoff, Locally original mathematics through writing, in Writing to Learn Mathematics and Science, P. Connolly and T. Vilardi, eds., Teachers College Press, New York, 1989, pp. 88-94.

[22] L. Baker and W. Saul, Considering science and language arts connections: a study of teacher cognition, J. Res. Sci. Teach. 31, 9 (1994), pp. 1023-1037.

[23] L. Cooley, Writing in calculus and reflective abstraction, J. Math. Beh. 21 (2002), pp. 255-282.

[24] W.J. Leonard, R.J. Dufresne and J.P. Mestre, Using qualitative problem-solving strategies to highlight the role of conceptual knowledge in solving problems, Am. J. Phys. 64, 12 (1996), pp. 1495-1503.

[25] J. Garofalo and F.K. Lester, Metacognition, cognitive monitoring, and mathematical performance, J. Res. Math. Ed. 16, 3 (1985), pp. 163-176.

[26] G.D. Gopen and D.A. Smith, What's an assignment like you doing in a course like this? Writing to learn mathematics, in Writing to Learn Mathematics and Science, P. Connolly and T. Vilardi, eds., Teachers College Press, New York, 1989, pp. 209-228.

[27] C.W.Keys, The development of scientific reasoning skills in conjunction with collaborative writing assignments: an interpretive study of six ninth-grade students, J. Res. Sci. Teach. 31, 9 (1994), pp. 1003-1022.

[28] D.N.M. Lew and H.G. Schmidt, Writing to learn: Can reflection journals be used to promote self-reflection and learning? High. Ed. Res. \& Dev. 30, 4 (2011), pp. 519-532.

[29] C. Morgan, Writing Mathematically: The Discourse of Investigation, Falmer Press, London, 1998.

[30] M. Nückles, S. Hübner, S. Dümer and A. Renki, Expertise reversal effects in writing-to-learn, Instr. Sci. 38 (2010), pp. 237-258.

[31] N.F. Ellerton and P.C. Clarkson, Language factors in mathematics teaching and learning, in International Handbook of Mathematics Education, A.J. Bishop et al., eds., Kluwer Academic Publishers, The Netherlands, 1996, pp. 987-1033.

[32] N.F. Ellerton and M.A.Clements, Researching language factors in mathematics education: the Australasian contribution, in Mathematics Education Research in Australasia 1992 - 1995, B. Atweh, K. Owens, P. Sullivan, eds., Mathematics Education Research Group of Australasia, Sydney, 1996, pp.191235.

[33] L.P. Rivard, A review of writing to learn in science: Implications for practice and research, J. Res. Sci. Teach. 31, 9 (1994), pp. 969-983.

[34] S. McCartney, Making better problem solvers through oral and written communication, Math. Middle Inst. Partn. Action Res. Proj. Univ. Nebraska (2009), pp. 1-36. Available at http://digitalcommons.unl.edu/cgi/viewcontent.cgi? article $=1038 \&$ context=mat hmidactionresearch, last accessed May 2011.

[35] T.S. Craig, Promoting Understanding in Mathematical Problem-solving through Writing: A Piagetian Analysis, PhD diss., University of Cape Town, 2007.

[36] T.S. Craig, Development of an engineering identity: Personal discovery of classroom mathematics in "real engineering", Afr. J. Res. Math. Sci. \& Tech. Ed. 14, 2 (2010), pp. 56-70. 